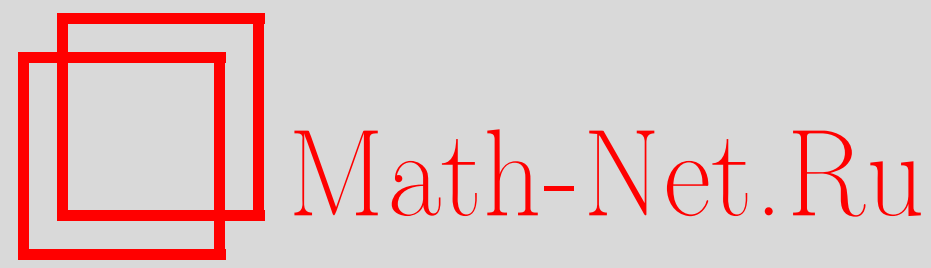

В. В. Шурыгин, Связность Эресмана для канонического слоения на многообразии над локальной алгеброй, Матем. заметки, 1996, том 59, выпуск 2, 303-310

DOI: https://doi.org/10.4213/mzm1717

Использование Общероссийского математического портала MathNet.Ru подразумевает, что вы прочитали и согласны с пользовательским соглашением

http://www . mathnet.ru/rus/agreement

Параметры загрузки:

IP : 54.157 .27 .8

26 апреля 2023 г., 14:46:25

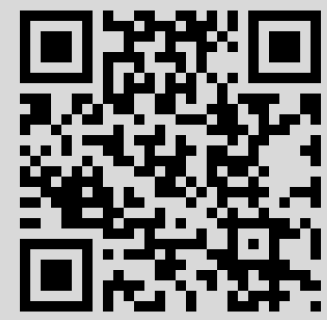


ТОм 59 выПУСК 2 фЕВРАЛ 1996

\section{СВЯЗНОСТЬ ЭРЕСМАНА ДЛЯ \\ КАНОНИЧЕСКОГО СЛОЕНИЯ НА \\ МНОГООБРАЗИИ НАД ЛОКАЛЬНОЙ АЛГЕБРОЙ}

В.В. Шурыгин

Введение. Многообразие $M^{\mathbb{A}}$ над локальной алгеброй $\mathbb{A}$ локально

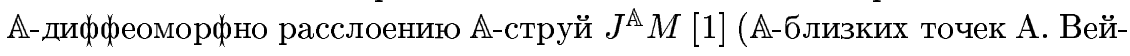
ля [2]). Это является причиной того, что уравнения горизонтального распределения $\mathbb{A}$-связности на $J^{\mathbb{A}} M$ сохраняют свой смысл и для многообразия $M^{\mathbb{A}}$, определяя на нем распределение, назьваемое $\mathbb{A}$-аффинным горизонтальным распределением. В случае алгебры дуальных чисел $\mathbb{R}(\varepsilon)$, например, это приводит к распределению на многообразии с интегрируемой почти-касательной структурой [3], заданному уравнениями горизонтального распределения аффинной связности на касательном расслоении [4]:

$$
d \dot{x}^{i}+\Gamma_{j k}^{i}\left(x^{m}\right) d x^{j} \dot{x}^{k}+t_{j}^{i}\left(x^{m}\right) d x^{j}=0 .
$$

В настоящей работе доказьвается, что $\mathbb{A}$-аффинное горизонтальное распределение на полном [5] многообразии $M^{\mathbb{A}}$ является связностью Эресмана в смысле Блюменталя-Хебды [6], [7], и что условием сушествования на $M^{\mathbb{A}} \mathbb{A}$-аффинного горизонтального распределения является обращение в нуль класса Атья [8] некоторого слоеного главного расслоения над $M^{\mathbb{A}}$.

1. Аффинные связности высших порядков. Мы будем использовать следующие обозначения (см. [1]): $\mathbb{R}(N, q)$ - алгебра срезанных многочленов от $N$ переменных степени $\leqslant q$ над полем $\mathbb{R}$ вещественных чисел. $\mathbb{A}=\mathbb{R}(N, q) / \mathbb{I}$ - локальная алгебра ширины $N$ и высоты $q, \AA$ максимальньй идеал алгебры $\mathbb{A}, \mathbb{A}^{n}$ - модуль строк длины $n$ с элементами из алгебры $\mathbb{A}, \AA^{n}$ - подмодуль в $\mathbb{A}^{n}$, состоящий из строк с элементами из $\stackrel{\AA}{\mathbb{A}}, \pi_{0}^{q}: \mathbb{A} \rightarrow \mathbb{R}$ и $\pi_{0}^{q}: \mathbb{A}^{n} \rightarrow \mathbb{R}^{n}$ - естественные эпиморфизмы. Модуль $\mathbb{A}^{n}$ (в частности, алгебру $\mathbb{A}$ ) можно представить в виде прямой суммы $\mathbb{A}^{n}=\mathbb{R}^{n}+\AA^{n}$. В соответствии с этим для произвольного элемента

(C) В.в. Шурыгин 1996 
$X \in \mathbb{A}^{n}$ получаем разложение $X=x+\stackrel{\circ}{X}$. Субмерсия $\pi_{0}^{q}: \mathbb{A}^{n} \rightarrow \mathbb{R}^{n}$ задает каноническое расслоение на $\mathbb{A}^{n}$, слои которого являются смежными классами по подмодулю $\AA^{n}$. Это слоение на $\mathbb{A}^{n}$ мы будем называть каноническим $\AA$-слоением. Для простоты будем предполагать все встречающиеся в дальнейшем дифференцируемые многообразия и отображения гладкими, т.е. класса $C^{\infty}$.

Гладкое отображение

$$
\varphi: U \subset \mathbb{A}^{n} \rightarrow U^{\prime} \subset \mathbb{A}^{n}
$$

назьвается $\mathbb{A}$-гладким, если касательное отображение $T_{x} \varphi$ является $\mathbb{A}$-линейньп при любом $X \in U$. $\mathbb{A}$-гладкое отображение $\varphi$ в простой [8] для

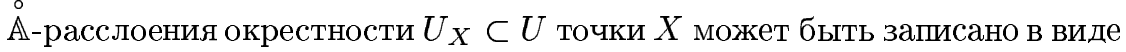

$$
X^{\prime i} \varphi^{i} \sum_{|p|=1}^{q} \frac{1}{p !} \frac{D^{p} \varphi^{i}}{D x^{p}} \stackrel{\circ}{X}^{p},
$$

где $i, j=1, \ldots, n, x^{i}=\pi_{0}^{q}\left(X^{i}\right), p=\left(p_{1}, \ldots, p_{n}\right)$ - мультииндекс длины $n$, а $\varphi^{i}: \pi_{0}^{q}\left(U_{X}\right) \rightarrow \mathbb{A}$ - некоторые $\mathbb{A}$-значные гладкие функции. Если отображение $\varphi$ задается уравнениями (3) на простой для $\AA$-слоения области $U$,

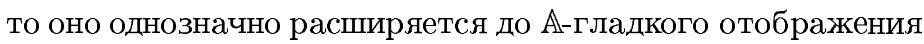

$$
\varphi:\left(\pi_{0}^{q}\right)^{-1}\left(\pi_{0}^{q}(U)\right) \rightarrow\left(\pi_{0}^{q}\right)^{-1}\left(\pi_{0}^{q}\left(U^{\prime}\right)\right),
$$

задаваемого той же формулой (3). При этом $\mathbb{A}$-диффеоморфизм расширяется до $\mathbb{A}$-диффеоморфизма. Если же область $U$ не является простой, то $\mathbb{A}$-гладкое отображение (2) может быть записано формулой (3), в которой $\varphi^{i}: U \rightarrow \mathbb{A}$ - базовые для $\mathbb{A}$-слоения функции.

Рассмотрим далее семейство диффеоморфизмов вида (4), обладающих свойством $\pi_{0}^{q}(\varphi(0))=0$. Каждый такой диффеоморфизм при ограничении на слой $\left(\pi_{0}^{q}\right)^{-1}(0)=\stackrel{\circ}{\mathbb{A}}^{n}$ определяет диффеоморфизм $\stackrel{\circ}{\varphi}: \stackrel{\circ}{\mathbb{A}}^{n} \rightarrow \stackrel{\circ}{\mathbb{A}}^{n}$ следующего вида

$$
\stackrel{\circ}{X}^{\prime i}=\sum_{|p|=1}^{q} \varphi_{p}^{i} \stackrel{\circ}{X}^{p}
$$

где $\varphi_{0}^{i} \in \stackrel{\circ}{\mathbb{A}}, \varphi_{p}^{i} \in \mathbb{A} / \AA^{q} q-|p|+1$ при $|p|>0$. Множество дифффеоморфизмов вида $(5)$ образуют группу Ли $D_{n}(\mathbb{A})$, назьваемую $\mathbb{A}$-аффинной группой [1]. Алгебру Ли группы $D_{n}(\mathbb{A})$ будем обозначать $d_{n}(\mathbb{A})$. Соотношениями $\varphi_{0}^{i}=0, \varphi_{p}^{i} \in \mathbb{R}$ в $D_{n}(\mathbb{A})$ выделяется подгруппа, изоморфная дифференщиальной группе $L_{n}^{q}[9]$. Если обозначить через $\varepsilon^{i}, i=1, \ldots, n$, стандартную систему образующих алгебры многочленов $\mathbb{R}(n, q)$, то элемент 
$Y$ из $D_{n}(\mathbb{A})$ может быть задан набором элементов

$$
Y^{i}=\sum_{|p|=1}^{q} \varphi_{p}^{i} \varepsilon^{p}
$$

из алгебры $\mathbb{A}(n, q)=\mathbb{A} \otimes \mathbb{R}(n, q) / \operatorname{Rad}(\mathbb{A} \otimes \mathbb{R}(n, q))^{q+1}$. Эти элементы будем назьвать $\mathbb{A}(n, q)$-координатами элемента $Y$. В дальнейшем будем иногда опускать знак суммы, если суммирование идет по индексам или мультииндексам, расположенным на разных уровнях. При этом композицию элементов $W=Z \circ Y$ в групе $D_{n}(\mathbb{A})$ будем записьвать в виде

$$
W^{i}=W_{p}^{i} \varepsilon^{p} Z_{r}^{i}\left(Y_{s}^{*} \varepsilon^{s}\right)^{r}=Z_{r}^{i} Y^{r}
$$

где $p, r, s$ - мультииндексы типа $\left(p_{1}, \ldots, p_{n}\right)$, изменяющиеся в пределах $0 \leqslant|p| \leqslant q$, а звездочкой обозначено место составляющих мультииндекca $r$.

Рассмотрим теперь некоторое расслоение $p: E \rightarrow M$ над $m$-мерным многообразием $M$ со стандартным слоем $\AA^{n}$ и структурной группой $D_{n}(\mathbb{A})$. На тотальном пространстве $E$ можно выбрать атлас тривиализации, области определения карт которого имеют вид $p^{-1}(U)$, где $U-$ область определения некоторой карты из гладкого атласа на $M$. При этом на $E$ получаются преобразования координат следующего вида

$$
x^{\alpha^{\prime}}=\varphi^{\alpha^{\prime}}\left(x^{\alpha}\right), \quad \stackrel{\circ}{X^{i^{\prime}}}=A_{p}^{i^{\prime}}\left(x^{\alpha}\right) \stackrel{\circ}{X^{p}},
$$

где $A_{p}^{i^{\prime}} \varepsilon^{p}-\mathbb{A}(n, q)$-координаты некоторого элемента из $D_{n}(\mathbb{A})$. Если в уравнениях (8) $n=m$ и $A_{p}^{i^{\prime}}=\frac{1}{p !} D^{p} \varphi^{i^{\prime}} / D x^{p}$, где функции $\varphi^{i^{\prime}}$ принимают значения в $\mathbb{R}$, то расслоение $E$ изоморфно расслоению $\mathbb{A}$-струй $J^{\mathbb{A}} M[1]$ (или расслоению $\mathbb{A}$-близких точек А. Вейля [2]). Преобразование координат (8) на $E$ порождает преобразование координат на тотальном пространстве $P$ главного расслоения $\rho: P \rightarrow M$, ассоциированного с $E$. Это преобразование имеет вид, аналогичный (8):

$$
x^{\alpha^{\prime}}=\varphi^{\alpha^{\prime}}\left(x^{\alpha}\right), \quad Y^{i^{\prime}}=A_{p}^{i^{\prime}}\left(x^{\alpha}\right) Y^{p}
$$

где $Y^{i}, Y^{i^{\prime}} \in \mathbb{A}(n, q)$. Расслоение $P$ будем назьвать расслоением $\mathbb{A}$-афффинных реперов расслоения $E$. В координатах правое действие $Z=R_{B} Y$ группы Ли $D_{n}(\mathbb{A})$ на $P$ имеет вид, аналогичньй $(7)$ :

$$
\left\{x^{\alpha}, Y^{i}\right\} \rightarrow\left\{x^{\alpha}, Z^{i}=Y_{p}^{i} B^{p}\right\}
$$


Рассмотрим далее связность $\Gamma$ в расслоении $P$. Элемент этой связности в точке $Y \in P$, рассматриваемый как 1-струя ростка сечения $\sigma:(M, x) \rightarrow(P, Y)$, определяется в координатах коэффициентами связности

$$
\Gamma_{\alpha p}^{i}(Y)=\left.\frac{\partial Y_{p}^{i}}{\partial x^{\alpha}}\right|_{x=\rho(Y)} .
$$

На координатной окрестности вида $\rho^{-1}(U)$ на $P$ локальное сечение $Y_{0}$ (поле $\mathbb{A}$-аффинных реперов) с уравнениями $Y_{p}^{i}\left(x^{\alpha}\right)=\delta_{p}^{i}$ будем называть натуральным. Здесь $\delta_{p}^{i}$ равняется 1 , когда на $i$-м месте мультииндекса $p$ стоит единица, на остальных - нули, и равняется 0 во всех других случаях. Коэффициенты связности $\Gamma$, вычисленные в точках натурального сечения, являются функциями $\Gamma_{\alpha p}^{i}=\Gamma_{\alpha p}^{i}\left(x^{\beta}\right)$. Будем называть эти функции коэффициентами $\mathbb{A}$-аффинной связности в данной локальной системе координат.

Связность в главном расслоении определяет горизонтальное распределение на всяком присоединенном расслоении [10]. Уравнения горизонтального распределения $\mathbb{A}$-аффинной связности на $E$ имеют вид

$$
d \stackrel{\circ}{X^{i}}-\Gamma_{\alpha p}^{i} \stackrel{\circ}{X^{p}} d x^{\alpha}=0 .
$$

Имеет место следующая

ТЕОРемА 1. При преобразованиях координат (8) коэффициенты $\mathbb{A}$-аффинной связности преобразуются по закону

$$
\Gamma_{\alpha^{\prime} r}^{i^{\prime}}\left(A_{s}^{*} \varepsilon^{s}\right)^{r}=\frac{\partial x^{\alpha}}{\partial x^{\alpha^{\prime}}} \partial_{\alpha} A_{p}^{i^{\prime}} \varepsilon^{p}+\frac{\partial x^{\alpha}}{\partial x^{\alpha^{\prime}}} A_{p}^{i^{\prime}} \sum_{i=1}^{n} p_{i} \Gamma_{\alpha s}^{i} \varepsilon^{p-i+s} .
$$

ДокАЗАТЕЛЬСтво. Дифференцируя (10), получим формулу, показывающую, как изменяются коэффициенты связности (9) при правом сдвиге $R_{B}$ :

$$
\Gamma_{\alpha p}^{i}(Z) \varepsilon^{p}=\Gamma_{\alpha s}^{i}(Y)\left(B_{r}^{*} \varepsilon^{r}\right)^{s} .
$$

Дифференцируя (8), получим формулу, связьвающую коэффициенты связности в точке $Y \in P$ в разных системах координат

$$
\Gamma_{\alpha^{\prime} r}^{i^{\prime}}(Y) \varepsilon^{p}=\partial_{\alpha} A_{p}^{i^{\prime}}\left(x^{\beta}\right) \frac{\partial x^{\alpha}}{\partial x^{\alpha^{\prime}}} Y^{p}+A_{p}^{i^{\prime}}\left(x^{\beta}\right) \sum_{i=1}^{n} P_{i} Y^{p-i} \Gamma_{\alpha s}^{i}(Y) \varepsilon^{s} \frac{\partial x^{\alpha}}{\partial x^{\alpha^{\prime}}}
$$

где обозначено $p-i=\left(p_{1}, \ldots, p_{i}-1, \ldots, p_{n}\right)$. Преобразование координат (9) индуцирует правый сдвиг натурального сечения $Y_{0} \rightarrow Y_{0}^{\prime}=R_{A} Y_{0}$, где элемент $A \in D_{n}(\mathbb{A})$ имеет координаты $A_{p}^{i^{\prime}}\left(x^{\alpha}\right)$ (см. (8)). При этом из $(14)$ следует

$$
\Gamma_{\alpha^{\prime} p}^{i^{\prime}}\left(Y_{0}\right) \varepsilon^{p}=\Gamma_{\alpha^{\prime} r}^{i^{\prime}}\left(Y_{0}^{\prime}\right)\left(A_{s}^{*} \varepsilon^{s}\right)^{r} .
$$

Подставляя (16) в (15), получаем (13). 


\section{2. Горизонтальные распределения на многообразиях над ло-}

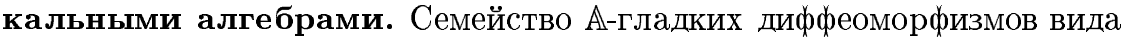
(2) образует псевдогруппу $\Gamma\left(\mathbb{A}^{n}\right)$. Многообразие $M^{\mathbb{A}}$ назьвается $\mathbb{A}$-гладким многообразием размерности $n$, если на $M^{\mathbb{A}}$ задан атлас с картами, принимающими значения в $\mathbb{A}^{n}$, и преобразованиями координат, принадлежащими псевдогруппе $\Gamma\left(\mathbb{A}^{n}\right)$. Такой атлас будем называть $\mathbb{A}$-атласом.

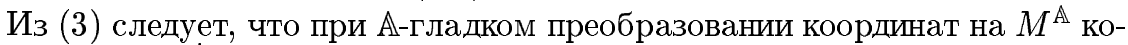
ординаты $x^{i^{\prime}}$ локально зависят только от координат $x^{i}$, таким образом, каноническое $\stackrel{\AA}{\mathbb{A}}$-слоение на $\mathbb{A}^{n}$ индуцирует слоение $\stackrel{\circ}{F}$ на $M^{\mathbb{A}}$, которое также будем назьвать каноническим ®-слоением.

ОПРЕДЕЛЕНИЕ. Предположим, что каждой карте $h: U \rightarrow \mathbb{A}^{n}$ из $\mathbb{A}$-атласа на $M^{\mathbb{A}}$ отнесен набор гладких $\mathbb{A}$-значных функций $\Gamma_{j p}^{i}$, заданных на области $h(U) \subset \mathbb{A}^{n}$ и являющихся базовыми относительно индуцированного $\mathbb{A}$-слоения. Предположим далее, что при $\mathbb{A}$-гладком преобразовании координат (3) эти функции преобразуются по закону $(13)$, где $A_{p}^{i^{\prime}}=\frac{1}{p !} D^{p} \varphi^{i^{\prime}} / D x^{p}$. Тогда на $M^{\mathbb{A}}$ уравнениями $($ см. $(12))$

$$
d \stackrel{\circ}{X}^{i}-\Gamma_{j p}^{i} \stackrel{\circ}{X}^{p} d x^{j}=0
$$

корректно определяется распределение $\nabla$ размерности $n$. Будем назьвать это распределение горизонтальньп $\mathbb{A}$-аффинным распределением.

Для исследования определенного вьше распределения нам потребуются некоторые расслоения над $M^{\mathbb{A}}$. Обозначим символом $O^{v} M^{\mathbb{A}}$ и назовем вертикальньп соприкасающимся расслоением многообразия $M^{\mathbb{A}}$ расслоение над $M^{\mathbb{A}}$ со стандартным слоем $\AA^{n}$ и функциями склейки, имеющими вид (3), над пересечением областей определения карт из $\mathbb{A}$-атласа. Если $p: O^{v} M^{\mathbb{A}} \rightarrow M^{\mathbb{A}}$ - каноническая проекция, а $h: U \rightarrow \mathbb{A}^{n}-\mathbb{A}$-карта на $M^{\mathbb{A}}$, относящая точке $X \in U$ координаты $\left\{x^{i}, \stackrel{\circ}{X}^{i}\right\}$, где $x^{i} \in \mathbb{R}, \stackrel{\circ}{X^{i}} \in \stackrel{\circ}{\mathbb{A}}$, то точка из $p^{-1}(U)$ в индуцированной на $O^{v} M^{\mathbb{A}}$ карте определяется координатами $\left\{x^{i}, \stackrel{\circ}{X^{i}}, \stackrel{*}{X^{i}}\right\}$ с $\stackrel{*}{X^{i}} \in \stackrel{\circ}{\mathbb{A}}$. Главное $D_{n}(\mathbb{A})$-расслоение, присоединенное к $O^{v} M^{\mathbb{A}}[10]$, обозначим через $P^{v} M^{\mathbb{A}}$ и назовем расслоением вертикальных соприкасающихся $\mathbb{A}$-аффинных реперов. Если в рассмотренной конструкции заменить в функциях склейки (3) $\varphi^{i}$ на $\bar{\varphi}^{i}=\pi_{0}^{q} \circ \varphi^{i}$, то получим, соответственно, расслоение трансверсальных $\mathbb{A}$-струй над $M^{\mathbb{A}}$ и расслоение трансверсальных $q$-реперов (см. [5], [8]). Все указанные расслоения принадлежат к типу слоеных расслоений [8], [11]. Если отнести каждой точке $X \in U \subset M^{\mathbb{A}}$ с координатами $\left\{x^{i}, \stackrel{\circ}{X}^{i}\right\}$ точку из слоя $p^{-1}(X) \subset O^{v} M^{\mathbb{A}}$ 
с координатами $\left\{x^{i}, \stackrel{\circ}{X^{i}}, \stackrel{*}{X^{i}}=\stackrel{\circ}{X^{i}}\right\}$, то корректно определится сечение

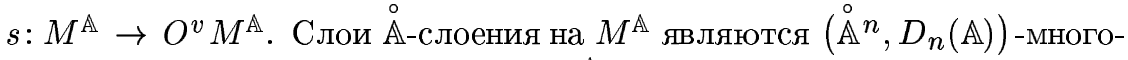
образиями [5], [12]. Многообразие $M^{\mathbb{A}}$ называется полным [5], если слои $\AA$-слоения на $M^{\mathbb{A}}$ являются полными как $\left(\AA^{n}, D_{n}(\mathbb{A})\right)$-многообразия [12], [5]. Для полного многообразия $M^{\mathbb{A}}$ можно определить проекцию $C: O^{v} M^{\mathbb{A}} \rightarrow M^{\mathbb{A}}$, которая в окрестности образа сечения $s\left(M^{\mathbb{A}}\right)$ имеет вид $C:\left\{x^{i}, \stackrel{\circ}{X^{i}}, \stackrel{*}{X^{i}}\right\} \rightarrow\left\{x^{i}, \stackrel{*}{X^{i}}\right\}$ и в ограничении на слой $p^{-1}(X)$ представляет собой накрытие $q_{X} \circ D_{X}^{-1}$, где $q_{X}: \widetilde{F}_{X} \rightarrow \stackrel{\circ}{F}_{X}-$ универсальное накрытие, а $D_{X}: \widetilde{F}_{X} \rightarrow p^{-1}(X)$ - развертьвающее отображение (см. [12], а также [13] для аффинных многообразий). Отметим, что сечение $s$ и отображение $C$ в более общей ситуации $(X, G)$-слоении рассматривались М.А. Махальцевым. Прообраз $T C^{-1}(\nabla) \mathbb{A}$-аффиинного горизонтального распределения $\nabla$ при проекции $C$ являются горизонтальным распределением проектируемой относительно поднятого слоения [8] (базовой [11]) связности в $O^{v} M^{\mathbb{A}}$. В локальных координатах распределение $T C^{-1}(\nabla)$ задается уравнениями

$$
d \stackrel{*}{X}^{i}-\Gamma_{j p}^{i} \stackrel{*}{X^{p}} d x^{j}=0 .
$$

Распределению $T C^{-1}(\nabla)$ на $O^{v} M^{\mathbb{A}}$ соответствует проектируемая связность $\widetilde{\nabla}$ в главном расслоении $P^{v} M^{\mathbb{A}}$. Необходимым и достаточным условием существования проектируемой связности в слоеном главном расслоении $P$ является обращение в нуль класса Атья $a(P)$ в когомологиях Молино [8] $H_{\text {adj } F}^{1,1}(P, \mathfrak{g})$, где $\mathfrak{g}$ - алгебра Ли структурной группы Ли $G$ расслоения $P$. Другие препятствия к существованию проектируемой связности были найдены в работе [11].

ТЕОремА 2. $\mathbb{A}$-аффинное горизонтальное распределение на полном $\mathbb{A}$-гладком многообразии $M^{\mathbb{A}}$ является связностью Эресмана в смысле Блюменталя-Хебды [6], [7]. $\mathbb{1}$-аффинное горизонтальное распределение на $M^{\mathbb{A}}$ существует тогда и только тогда, когда класс Атья $a\left(P^{v} M^{\mathbb{A}}\right) \in H_{\operatorname{adj} F}^{1,1}\left(P^{v} M^{\mathbb{A}}, d_{n}(\mathbb{A})\right)$ обрашается в нуль.

ДокАЗАТЕЛьСТво. Горизонтальное распределение на многообразии $M$ со слоением $F$ назьвается связностью Эресмана [6], [7], если для произвольной пары, состоящей из горизонтальной кусочно-гладкой кривой $\sigma:[0,1] \rightarrow M$ и вертикальной (т.е. лежащей в слое слоения $F$ ) кусочногладкой кривой $\tau:[0,1] \rightarrow M$ с началом в одной и той же точке $X_{0}=$ $\sigma(0)=\tau(0)$ существует кусочно-гладкое отображение $\delta:[0,1] \times[0,1] \rightarrow M$ такое, что ограничение $\delta: a_{0} \times[0,1] \rightarrow M$ является горизонтальной кривой при всяком $a_{0}$ и совпадает с $\sigma$ при $a_{0}=0$, а ограничение $\delta:[0,1] \times b_{0} \rightarrow M$ является вертикальной кривой при всяком $b_{0}$ и совпадает с $\tau$ при $b_{0}=0$. 
Рассмотрим на многообразии $M^{\mathbb{A}}$ горизонтальную кривую $\sigma$ и вертикальную кривую $\tau$ с обшим началом $X_{0}=\sigma(0)=\tau(0)$. По определению распределения $T C^{-1}(\nabla)$ кривая $\widetilde{\sigma}=s \circ \sigma$ является горизонтальной кривой для $\mathbb{A}$-аффинной связности на $O^{v} M^{\mathbb{A}}$. Для кривой $\tau$ существует единственная кривая $\widetilde{\tau}$ на слое $p^{-1}\left(X_{0}\right)$, переходящая в $\tau$ при отображении $C$ (накрывающая кривая). При этом $\widetilde{\tau}(0)=s(\tau(0))=\widetilde{\sigma}(0)$. Перенося параллельно точки $\tau\left(a_{0}\right) \in p^{-1}\left(X_{0}\right), a_{0} \in[0,1]$, вдоль $\sigma$, получим отображение $\widetilde{\delta}:[0,1] \times[0,1] \rightarrow O^{v} M^{\mathbb{A}}$. Композиция $\delta=C$ ॰ $\widetilde{\delta}:[0,1] \times[0,1] \rightarrow M^{\mathbb{A}}$ удовлетворяет требованиям определения Блюменталя-Хебды.

Поскольку горизонтальное распределение проектируемой связности в расслоении $O^{v} M^{\mathbb{A}}$ над полным $M^{\mathbb{A}}$ переходит при отображении $C$ в $\mathbb{A}$-аффинное горизонтальное распределениена $M^{\mathbb{A}}$, то $\mathbb{A}$-аффинное горизонтальное распределение на $M^{\mathbb{A}}$ существует тогда и только тогда, когда существует проектируемая связность в $P^{v} M^{\mathbb{A}}$. Отсюда следует второе утверждение теоремы для полного многообразия $M^{\mathbb{A}}$. Однако, поскольку для определения и $\mathbb{A}$-аффинного горизонтального распределения и связности в $P^{v} M^{\mathbb{A}}$ достаточно задания базовых функций $\Gamma_{j k}^{i}$ (см. (17) и (18)), это утверждение справедливо и для произвольного $\mathbb{A}$-гладкого многообразия $M^{\mathbb{A}}$.

Замечания. 1. Из результатов работы [6] следует, что если неполное связное многообразие $M^{\mathbb{A}}$ допускает $\mathbb{A}$-аффинное горизонтальное распределение, являюшееся связностью Эресмана, то универсальные накрывающие слоев канонического $\AA$-слоения являются изоморфными $\left(\AA^{n}, D_{n}(\mathbb{A})\right)$-многообразиями.

2 . Если $a\left(P^{v} M^{\mathbb{A}}\right) \neq 0$, то многообразие $M^{\mathbb{A}}$ не может быть $\mathbb{A}$-диффеоморфно никакому расслоению $J^{\mathbb{A}} M$.

В заключение приведем пример многообразия над алгеброй $\mathbb{R}(\varepsilon)$, не допускающего аффинного горизонтального распределения. Для этого на плоскости $\mathbb{R}(\varepsilon)$ дуального переменного $X=x+\varepsilon \dot{x}$ рассмотрим полосу $0<\dot{x}<\lambda^{\prime}(x)+\alpha$, где $\lambda^{\prime}(x)=\beta(\operatorname{arctg}(x+\gamma)-\operatorname{arctg} \gamma)+1$, а $\alpha, \beta$ и $\gamma$ - достаточно малые положительные числа, например, $\alpha=\beta=\gamma=0.01$. Отождествляя точки $y+\varepsilon \dot{y}$ и $x+\varepsilon \dot{x}$ этой полосы при $y=\lambda(x), \dot{y}=\dot{x} \lambda^{\prime}(x)+\lambda^{\prime}(x)$, где $\lambda(x)=\int_{0}^{x} \lambda^{\prime}(x) d x$, получим цилиндр, которьй наследует структуру многообразия над алгеброй $\mathbb{R}(\varepsilon)$. Предполагая, что на этом цилиндре имеется аффинное горизонтальное распределение, определяемое коэффищиентами $\Gamma$ и $t$ (см. (1)), при $x=0$ получим противоречие с законом преобразования коэффициента $\Gamma: \Gamma(\lambda(x))=\Gamma(x) \lambda^{\prime}(x)+\lambda^{\prime \prime}(x) / \lambda^{\prime}(x)$, поскольку $\lambda(0)=0, \lambda^{\prime}(0)=1, \lambda^{\prime \prime}(0) \neq 0$. 


\section{СПИСОК ЦИТИРОВАННОЙ ЛИТЕРАТУРЫ}

[1] Шурыгин В.В. Расслоения струй как многообразия над алгебрами // Проблемы геометрии. Итоги науки и техники. Т. 19. М.: ВИНИТИ, 1987. C. 3-22.

[2] Weil A. Théorie des points proches sur les variétés différentiales // Colloq. Internat. Centre Nat. Rech. Sci. V. 52. Strasburg, Paris, 1953. P. 111-117.

[3] Brickell F., Clark R.S. Integrable almost tangent structures // J. Differ. Geom. 1974. V. 9. № 4. P. 557-563.

[4] Веблен О., Уайтхед Дж. Основания дифференциальной геометрии. М.: ИЛ, 1949.

[5] Шурыгин В. В. Многообразия над локальными алгебрами, эквивалентные расслоениям струй // Изв. вузов. Матем. 1992. №10. С. 68-79.

[6] Blumental R. A., Hebda J. J. Ehresmann connections for foliations // Indiana Math. J. 1984. V. 33. № 4. P. 597-612.

[7] Blumental R. A., Hebda J. J. Complementary distributions which preserve the leaf geometry and applications to totally geodesic foliations // Quart. J. Math. 1984. V. 35. P. 383-392.

[8] Molino P. Riemannian foliations. Boston-Basel: Birkhäuser, 1988.

[9] Евтушик Л.Е., Лумисте Ю. Г., Остиану Н. М., Широков А. П. Дифференциально-геометрические структуры на многообразиях // Проблемы геометрии. Итоги науки и техники. Т. 9. М.: ВИНИТИ, 1979.

[10] Кобаяси Ш., Номидзу К. Основы дифференциальной геометрии. Т. 1. M.: Наука, 1981.

[11] Kamber F. W., Tondeur P. Foliated bundles and characteristic classes // Lecture Notes in Math. 1975. № 493.

[12] Апанасов Б. Н. Геометрия дискретных многообразий. М.: Наука, 1991.

[13] Fried D., Goldman W., Hirsch M. W. Affine manifolds with nilpotent holonomy // Comment. Math. Helv. 1981. V. 56. № 4. P. 487-523. 